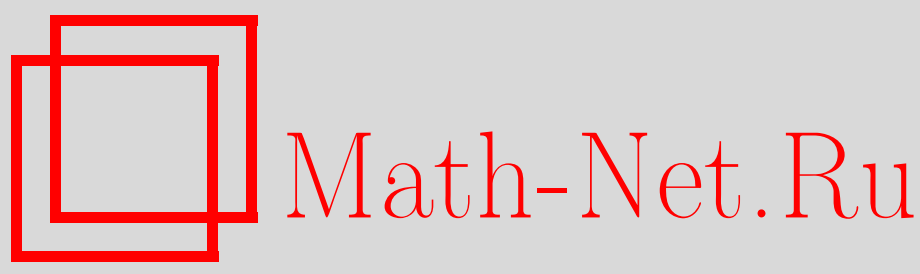

А. Г. Петров, О параметрических представлениях ортогональных и симплектических матриц, Изв. вузов. Матем., 2020, номер 6, 93-98

DOI: https://doi.org/10.26907/0021-3446-2020-6-93-98

Использование Общероссийского математического портала Math-Net.Ru подразумевает, что вы прочитали и согласны с пользовательским соглашением

http://www . mathnet.ru/rus/agreement

Параметры загрузки:

IP : 107.22 .136 .117

26 апреля 2023 г., 08:41:15 
Краткое сообщение, представленное Д.В. Маклаковым

\title{
А.Г. ПЕТРОВ
}

\section{О ПАРАМЕТРИЧЕСКИХ ПРЕДСТАВЛЕНИЯХ ОРТОГОНАЛЬНЫХ И СИМПЛЕКТИЧЕСКИХ МАТРИЦ}

\begin{abstract}
Аннотация. Симплектические матрицы подчинены определенным условиям, которым удовлетворяют якобиевые матрицы преобразований, сохраняющих гамильтонову форму дифференциальных уравнений. Выведена формула, параметризующая симплектические матрицы симметричными матрицами. Проведена аналогия между полученной формулой и формулой Кэли, связывающей ортогональные и антисимметричные матрицы. Показано, что ортогональные и антисимметричные матрицы при замене декартовой системы координат преобразуются по ковариантному закону. Аналогично доказана ковариантность преобразований симплектических и симметричных матриц.

Из формул Кэли и их аналога получен ряд матричных соотношений, связывающих ортогональные и симметричные матрицы, и аналогичных соотношений, связывающих симплектические и симметричные матрицы.
\end{abstract}

Ключевые слова: симплектическая и симметричная матрица, ортогональная и антисимметричная матрица, ковариантность.

УДК: 512.643

DOI: $10.26907 / 0021-3446-2020-6-93-98$

1. Параметрическое представление ортогональных матриц. Для матриц, удовлетворяющих условию ортогональности

$$
O O^{T}=O^{T} O=E
$$

известны формулы Кэли [1]:

$$
O=(E-K)(E+K)^{-1}, \quad K=(E-O)(E+O)^{-1} .
$$

Первая выражает ортогональную матрицу $O$ через антисимметричную матрицу $K^{T}=-K$. Здесь $E$ - единичная (тождественная) матрица, верхний индекс $T$ означает знак транспонирования. При любой антисимметричной матрице $K$ матрица, определенная по первой формуле (2), удовлетворяет условию ортогональности $O O^{T}=E$.

Вторая формула (2) является обращением первой формулы и выражает матрицу $K$ через ортогональную матрицу $O$. Для любой ортогональной матрицы $O$, собственное число которой не равно -1 , матрица $K$ во второй формуле $(2)$ удовлетворяет условиям антисимметрии.

Поступила в редакцию 24.03.2020, после доработки 24.03.2020. Принята к публикации 25.03.2020.

Благодарности. Работа выполнена в рамках госзадания (номер госрегистрации АAAA-A20120011690138-6). 
В трехмерном пространстве ортогональные матрицы размера $3 \times 3$ играют важную роль в теории навигации (определение положения твердого тела в пространстве [2], [3]). В этом случае матрица $K$ в формулах (2) параметризует ортогональную матрицу, выражая все ее девять элементов через независимые три элемента матрицы $K$. Такая параметризация имеет преимущества перед параметризацией через углы Эйлера из-за известной неопределенности при угле нутации равном нулю. Существуют и другие способы параметризации ортогональной матрицы через антисимметричную [2].

Из равенства $O O^{T}=E$ следует, что определитель ортогональной матрицы равен 1 или -1. Для ортогональных матриц с определителем $\operatorname{det}(O)=1$ из первой формулы Кэли (2) следует

$$
\operatorname{det}(E+K)=\operatorname{det}(E-K)
$$

С помощью тождеств

$$
(E-O)(E+O)=(E+O)(E-O), \quad(E-K)(E+K)=(E+K)(E-K)
$$

формулы Кэли (2) можно записать двумя способами:

$$
\begin{gathered}
O=(E-K)(E+K)^{-1}=(E+K)^{-1}(E-K), \\
K=(E-O)(E+O)^{-1}=(E+O)^{-1}(E-O) .
\end{gathered}
$$

Отсюда следует

$$
\operatorname{det}(K)=\operatorname{det}(E-O) / \operatorname{det}(E+O) .
$$

Преобразуя с помощью первой формулы Кэли (2)

$$
E+O=E-(E+K-2 E)(E+K)^{-1}=2(E+K)^{-1}
$$

и используя равенства определителей (3), получаем формулы

$$
(E+O)(E+K)=2 E \Rightarrow \operatorname{det}(E+O) \operatorname{det}(E \pm K)=2^{2 n} .
$$

Представляет интерес найти закон преобразования матриц $O$ и $K$ при переходе из одной декартовой системы в другую. Такой переход можно осуществить с помощью ортогональной матрицы $C$. Пусть матрица $O$ определяет преобразование радиус-вектора $\mathbf{r}$ в радиус-вектор $\mathbf{R}=O \mathbf{r}$. В новой системе координат имеем $\mathbf{r}^{\prime}=C \mathbf{r}, \quad \mathbf{R}^{\prime}=C \mathbf{R}$. Чтобы найти новую матрицу $O^{\prime}$ преобразования $\mathbf{R}^{\prime}=O^{\prime} \mathbf{r}^{\prime}$, запишем это преобразование в исходной системе координат

$$
C \mathbf{R}=O^{\prime} C \mathbf{r} \Rightarrow \mathbf{R}=C^{T} O^{\prime} C \mathbf{r} .
$$

Отсюда следует закон преобразования

$$
O=C^{T} O^{\prime} C \Rightarrow O^{\prime}=C O C^{T} .
$$

Соответствующий закон преобразования для матрицы $K$ в формуле Кэли вытекает из следующей группы матричных преобразований

$$
\begin{aligned}
& O^{\prime}=C O C^{T} \Rightarrow\left(E+K^{\prime}\right)^{-1}\left(E-K^{\prime}\right)=C(E-K)(E+K)^{-1} C^{-1} \Rightarrow \\
& \left(E-K^{\prime}\right) C(E+K)=\left(E+K^{\prime}\right) C(E-K) \Rightarrow \\
& C(E+K-E+K)=K^{\prime} C(E+K+E-K) \Rightarrow 2 C K=2 K^{\prime} C \Rightarrow \\
& \Rightarrow K^{\prime}=C K C^{T} \text {. }
\end{aligned}
$$

Таким образом, преобразования матриц (9) и (10) ковариантны, иначе говоря, матрицы $O$ и $K$ в формулах Кэли преобразуются по одному и тому же закону. 
2. Параметрическое представление симплектических матриц. Матрица $A$ размерности $2 n \times 2 n$ называется симплектической, если она удовлетворяет соотношению

$$
A I A^{T}=A^{T} I A=I, \quad I=\left(\begin{array}{cc}
0 & E_{n} \\
-E_{n} & 0
\end{array}\right),
$$

где $E_{n}-$ единичная матрица размерности $n \times n$.

Матрица $I$ называется симплектической единицей. Она является симплектической и обладает свойством $I I=I^{2}=-E$.

Условие симплектичности (11) является аналогом условия ортогональности (1). Так же, как для ортогональной матрицы, определитель симплектической матрицы равен либо 1 , либо -1. Для определенности здесь рассматриваются симплектические матрицы с определитем равным 1.

Симплектические матрицы используются в гамильтоновой механике. Такой матрицей является якобиева матрица преобразования системы дифференциальных уравнений, сохраняющая гамильтонову форму [3]. Можно провести аналогию между формулами (1)-(10) и соответствующими формулами для симплектических матриц. Для этого надо поставить в соответствие ортогональной матрице $O$ симплектическую $A$ и антисимметричной $-K$ произведение матрицы $I$ и симметричной матрицы $\frac{1}{2} \Psi\left(\Psi^{T}=\Psi\right)$. Кратко это соответствие записывается так:

$$
O \rightarrow A, \quad K \rightarrow \frac{1}{2} I \Psi
$$

Теорема 1. Формулам Кэли (2) соответствуют формуль

$$
A=\left(E+\frac{1}{2} I \Psi\right)\left(E-\frac{1}{2} I \Psi\right)^{-1}, \quad \frac{1}{2} I \Psi=(E+A)^{-1}(A-E) .
$$

1) Если $\Psi^{T}=\Psi$, то матрица A, определенная по первой формуле (12), симплектична.

2) Если матрича А симплектична, то матрица $\frac{1}{2} I \Psi$, определенная по второй формуле (12), удовлетворяет условию $\left(\frac{1}{2} I \Psi\right)^{T}=-\frac{1}{2} \Psi I$, т.е. матрица $\Psi$ симметрична.

Схема доказательства. 1) Из условия $\Psi^{T}=\Psi$ следует равенство $(I \Psi)^{T}=-\Psi I$. Отсюда для транспонированной матрицы (12) имеем $A^{T}=\left(E-\frac{1}{2}(I \Psi)^{T}\right)^{-1}\left(E+\frac{1}{2}(I \Psi)^{T}\right)=(E+$ $\left.\frac{1}{2} \Psi I\right)^{-1}\left(E-\frac{1}{2} I \Psi I\right)$. Подставив это выражение в формулу $A^{T} I A$, получим

$$
A^{T} I A=\left(E+\frac{1}{2} \Psi I\right)^{-1}\left(E-\frac{1}{2} \Psi I\right) I\left(E+\frac{1}{2} I \Psi\right)\left(E-\frac{1}{2} I \Psi\right)^{-1} .
$$

С помощью тождества, аналогичного (4),

$$
\left(E-\frac{1}{2} \Psi I\right) I\left(E+\frac{1}{2} I \Psi\right)=\left(E+\frac{1}{2} \Psi I\right) I\left(E-\frac{1}{2} I \Psi\right)
$$

получим $A^{T} I A=I$.

Для доказательства утверждения 2) применяется

Лемма.

$$
E+I A^{-1} I=I(E-A) A^{-1} I, \quad E-I A^{-1} I=I(-E-A) A^{-1} I .
$$

Транспонируем второе равенство теоремы из (12), используя соотношение $A^{T}=-I A^{-1} I$, вытекающее из условия симплектичности, и преобразуем его с помощью леммы:

$$
\left(\frac{1}{2} I \Psi\right)^{T}=-\frac{1}{2} \Psi^{T} I=-\left(E+I A^{-1} I\right)\left(E-I A^{-1} I\right)^{-1}=I(E-A) A^{-1} I I A(E+A)^{-1} I=-\frac{1}{2} \Psi I .
$$


Из полученного равенства следует $-\Psi^{T} I=-\Psi I \Rightarrow \Psi^{T}=\Psi$, что и требовалось доказать.

Для симплектических матриц с определителем $\operatorname{det}(A)=1$ из первой формулы $(12)$ вытекает

$$
\operatorname{det}\left(E+\frac{1}{2} I \Psi\right)=\operatorname{det}\left(E-\frac{1}{2} I \Psi\right)
$$

С помощью тождеств, аналогичных (4),

$$
(E-A)(E+A)=(E+A)(E-A), \quad\left(E-\frac{1}{2} I \Psi\right)\left(E+\frac{1}{2} I \Psi\right)=\left(E+\frac{1}{2} I \Psi\right)\left(E-\frac{1}{2} I \Psi\right)
$$

формулы (12) можно записать двумя способами:

$$
\begin{gathered}
A=\left(E+\frac{1}{2} I \Psi\right)\left(E-\frac{1}{2} I \Psi\right)^{-1}=\left(E-\frac{1}{2} I \Psi\right)^{-1}\left(E+\frac{1}{2} I \Psi\right), \\
\frac{1}{2} I \Psi=-(E+A)^{-1}(E-A)=-(E-A)(E+A)^{-1} .
\end{gathered}
$$

Из (15) следует

$$
\operatorname{det}\left(\frac{1}{2} I \Psi\right)=-\operatorname{det}(E-A) / \operatorname{det}(E+A)
$$

Преобразуя с помощью аналога первой формулы Кэли (12)

$$
E+A=E-\left(E-\frac{1}{2} I \Psi-2 E\right)\left(E-\frac{1}{2} I \Psi\right)^{-1}=2\left(E-\frac{1}{2} I \Psi\right)^{-1}
$$

и используя равенства определителей (13), получаем формулы

$$
(E+A)\left(E-\frac{1}{2} I \Psi\right)=2 E, \quad \operatorname{det}(E+A) \operatorname{det}\left(E \pm \frac{1}{2} I \Psi\right)=2^{2 n} .
$$

Так же, как для закона преобразования матриц $O$ и $K$, при переходе из одной декартовой системы в другую найдем закон преобразования матриц $A$ и $\Psi$. Такой переход можно осуществить с помощью симплектической матрицы $B, B I B^{T}=I$.

Пусть симплектическая матрица $A$ определяет преобразование радиус-вектора $\mathbf{r}$ в радиусвектор $\mathbf{R}=A \mathbf{r}$. В новой системе координат имеем $\mathbf{r}^{\prime}=B \mathbf{r}, \quad \mathbf{R}^{\prime}=B \mathbf{R}$. Чтобы найти новую матрицу $A^{\prime}$ преобразования $\mathbf{R}^{\prime}=A^{\prime} \mathbf{r}^{\prime}$, запишем это преобразование в исходной системе координат

$$
B \mathbf{R}=A^{\prime} B \mathbf{r} \Rightarrow \mathbf{R}=B^{-1} A^{\prime} B \mathbf{r} .
$$

Отсюда следует закон преобразования

$$
A=B^{-1} A^{\prime} B \Rightarrow A^{\prime}=B A B^{-1} .
$$

Соответствующий закон преобразования для матрицы $\Psi$ в формулах теоремы 1 вытекает из матричных преобразований с помощью (15)

$$
\begin{gathered}
A^{\prime}=B A B^{-1} \Rightarrow\left(E-\frac{1}{2} I \Psi^{\prime}\right)^{-1}\left(E+\frac{1}{2} I \Psi^{\prime}\right)=B\left(E+\frac{1}{2} I \Psi\right)\left(E-\frac{1}{2} I \Psi\right)^{-1} B^{-1} \Rightarrow \\
\left(E+\frac{1}{2} I \Psi^{\prime}\right) B\left(E-\frac{1}{2} I \Psi\right)=\left(E-\frac{1}{2} I \Psi^{\prime}\right) B\left(E+\frac{1}{2} I \Psi\right) \Rightarrow \\
B\left(E-\frac{1}{2} I \Psi-E-\frac{1}{2} I \Psi\right)=\frac{1}{2} I \Psi^{\prime} B\left(-E+\frac{1}{2} I \Psi-E-\frac{1}{2} I \Psi\right) \Rightarrow B I \Psi=I \Psi^{\prime} B \Rightarrow \\
I \Psi^{\prime}=B I \Psi B^{-1} .
\end{gathered}
$$

Таким образом, преобразования матриц (19) и (20) ковариантны, иначе говоря, матрицы (19) и (20) в формуле (12) преобразуются по одному и тому же закону. Этот результат назовем теоремой о ковариантности параметризации симплектической матрицы. Он аналогичен теореме о ковариантности формулы Кэли для ортогональной матрицы. 
Теорема 2. Пусть симплектическая матрица $A$, параметризуемая симметричной матрицей $\Psi$ по первой формуле (12), определяет отображение $\mathbf{R}=A \mathbf{r}$. Тогда это отображение при любом симплектическом преобразовании $\mathbf{r}^{\prime}=B \mathbf{r}, \mathbf{R}^{\prime}=B \mathbf{R}, B^{T} I B=I$ представится в виде

$$
\mathbf{R}^{\prime}=A^{\prime} \mathbf{r}^{\prime}, \quad A^{\prime}=\left(E+\frac{1}{2} I \Psi^{\prime}\right)\left(E-\frac{1}{2} I \Psi^{\prime}\right)^{-1},
$$

в котором матрицы $A$ и $I \Psi$ преобразуются по одинаковому закону

$$
A^{\prime}=B A B^{-1}, \quad I \Psi^{\prime}=B I \Psi B^{-1} .
$$

При этом матрица $A^{\prime}$ останется симплектической, а матрица $\Psi^{\prime}$ симметричной.

3. Параметризация симплектических преобразований. В [4] введена параметрическая форма замены переменных $(\mathbf{q}, \mathbf{p} \rightarrow \mathbf{Q}, \mathbf{P})$

$$
\begin{array}{ll}
\mathbf{q}=\mathbf{x}-\frac{1}{2} \Psi_{\mathbf{y}}, & \mathbf{Q}=\mathbf{x}+\frac{1}{2} \Psi_{\mathbf{y}}, \\
\mathbf{p}=\mathbf{y}+\frac{1}{2} \Psi_{\mathbf{x}}, & \mathbf{P}=\mathbf{y}-\frac{1}{2} \Psi_{\mathbf{x}},
\end{array}
$$

где $\Psi(t, \mathbf{x}, \mathbf{y})$ - дважды непрерывно дифференцируемая функция.

Якобиан преобразования $A=\frac{\partial(\mathbf{Q}, \mathbf{P})}{\partial(\mathbf{q}, \mathbf{p})}$ имеет вид

$$
A=\left(E+\frac{1}{2} I \Psi\right)\left(E-\frac{1}{2} I \Psi\right)^{-1}, \quad \Psi=\left(\begin{array}{ll}
\Psi_{\mathrm{xx}} & \Psi_{\mathrm{xy}} \\
\Psi_{\mathrm{xy}} & \Psi_{\mathrm{yy}}
\end{array}\right)
$$

совпадающий с формулой (12), у которой матрица $\Psi$ является симметричной матрицей гессиана функции $\Psi(t, \mathbf{x}, \mathbf{y})$. По теореме 1 эта матрица симплектична и по локальному критерию каноничности преобразование (21) сохраняет гамильтонову форму уравнений. В этом преобразовании функция $\Psi(t, \mathbf{x}, \mathbf{y})$ является производящей функцией.

На "удручающую неинвариантность производящих функций Якоби относительно выбора системы координат" неоднократно обращается внимание в монографии [5]. Теорема 2 доказывает, что введенная параметрическая производящая функция ковариантна по отношению к любой симплектической замене переменных, и этим она выгодно отличается от традиционных производящих функций Якоби.

4. Выводы. С помощью простых матричных преобразований выведены соотношения, связывающие симплектические и симметричные матрицы (11)-(20). Они аналогичны соотношениям, связывающих ортогональные и антисимметричные матрицы (1)-(10).

Формулы Кэли упрощают преобразование ортогональных матриц $O \rightarrow O^{\prime}$. Пусть матрица $O$ выражается через антисимметричную матрицу

$$
K=\left(\begin{array}{ccc}
0 & k_{3} & -k_{2} \\
-k_{3} & 0 & k_{1} \\
k_{2} & -k_{1} & 0
\end{array}\right), \quad \mathbf{k}=\left(\begin{array}{c}
k_{1} \\
k_{2} \\
k_{3}
\end{array}\right),
$$

по формуле Кэли (2). При ортогональной замене с матрицей $C$ формулу преобразования можно заменить на преобразование вектора $\mathbf{k}^{\prime}=C \mathbf{k}$, выразить через вектор $\mathbf{k}^{\prime}$ антисимметричную матрицу $K^{\prime}$ и по формуле Кэли найти преобразованную ортогональную матрицу $O^{\prime}=\left(E-K^{\prime}\right)\left(E+K^{\prime}\right)^{-1}$. Аналогично можно преобразовывать симплектические матрицы

$$
A=\left(E+\frac{1}{2} I \Psi\right)\left(E-\frac{1}{2} I \Psi\right)^{-1}
$$

выражая их через симметричную $\Psi$. 
Формулу преобразования $A^{\prime}=B A B^{-1}$, где $B$ - произвольная симплектическая матрица, можно заменить на следующую последовательность преобразований:

$$
I \Psi^{\prime}=B I \Psi B^{-1}, \quad A^{\prime}=\left(E+\frac{1}{2} I \Psi^{\prime}\right)\left(E-\frac{1}{2} I \Psi^{\prime}\right)^{-1} .
$$

Представляют интерес также тождества для определителей: (3), (8), (13), (18).

Кроме того, для трехмерных матриц из формул Кэли (2) следует

$$
\operatorname{det}(E \pm K)=1+\mathbf{k}^{2}, \quad \operatorname{det}(E-O)=\operatorname{det}(K)=0 .
$$

Последнее равенство означает, что собственное число ортогональной матрицы в трехмерном пространстве равно единице [3].

\title{
ЛИТЕРАТУРА
}

[1] Гантмахер Ф.Р. Теория матрии (М., Наука, М., 1967).

[2] Челноков Ю.Н. Кватернионные и бикватернионные модели и методы механики твердого тела и их приложения (ФИЗМАТЛИТ, М., 2006).

[3] Журавлев В.Ф. Основы теоретической механики (Наука, М., 1997).

[4] Петров А.Г. Асимптотические методы решения уравнениях Гамильтона с помощъю параметризации канонических преобразований, Диф. уравнения. 40 (5), 626-633 (2004).

[5] Арнольд В.И. Математические методь классической механики (Эдиториал УРСС, М., 2000).

\section{Александр Георгиевич Петров}

Институт проблем механики им. А.Ю. Ишлинского РАН, просп. Вернадского, 101, корп. 1, г. Москва, 119526, Россия,

e-mail : petrovipmech@gmail.com

\section{A.G.Petrov}

\section{On parametric representations of orthogonal and symplectic matrices}

\begin{abstract}
Symplectic matrices are subject to certain conditions that are inherent to the Jacobian matrices of transformations preserving the Hamiltonian form of differential equations. A formula is derived that parameterizes symplectic matrices with symmetric matrices. An analogy is drawn between the obtained formula and the Cayley formula that connects orthogonal and antisymmetric matrices. It is shown that orthogonal and antisymmetric matrices are transformed by the covariant law when replacing the Cartesian coordinate system. Analogously, the covariance of transformations of symplectic and symmetric matrices is proved.
\end{abstract}

Keywords: symplectic and symmetric matrixes, orthogonal and antisymmetric matrixes, covariance.

Alexander Georgievich Petrov

Ishlinsky Institute for Problems in Mechanics of the Russian Academy of Sciences, 101/1 pr. Vernadskogo, Moscow, 119526 Russia,

e-mail : petrovipmech@gmail.com 